\title{
Extrasolar planets and star formation: science opportunities for future ELTs
}

\author{
Karl R. Stapelfeldt \\ Jet Propulsion Laboratory, California Institute of Technology \\ Mail Stop 183-900, 4800 Oak Grove Drive, Pasadena, CA 91109 USA \\ email: krs@exoplanet.jpl.nasa.gov
}

\begin{abstract}
Future extremely large telescopes will be the engines of major progress in the fields of star and planet formation, brown dwarfs, and extrasolar planets. Their throughput will enable spectroscopic studies of the structure of brown dwarf atmospheres; reveal the composition and kinematics of protoplanetary disks; extend radial velocity searches for extrasolar planets to fainter stars and lower masses; and characterize the surfaces of the most distant Kuiper Belt objects. Their resolution will allow us to resolve and track the orbits of close binary young stars and brown dwarfs, establishing their dynamical masses and calibrating their evolutionary tracks; probe the inner region of young stellar object disks, resolving the jet collimation region in accreting systems and disk inner holes in more evolved ones; and move the horizon for stellar companion searches inward to 40 mas, enabling the direct detection of hot young planets orbiting $10 \mathrm{AU}$ from young $\mathrm{T}$ Tauri stars. The high contrast imaging capability of future ELTs is uncertain, and depends on future developments in extreme adaptive optics. Wavefront control considerations suggest a fundamental atmospheric contrast limit of $10^{-8}$ for companion searches to nearby solar-type stars, below which detections are unlikely to be possible. To reach this performance level, continuing investments will be needed in extreme adaptive optics work, in addition to careful attention to the specialized requirements of ultra-high contrast imaging in ELT design. Above the $10^{-8}$ contrast limit, a $30 \mathrm{~m}$ telescope has the potential to directly image about a dozen of the currently known radial velocity planets.
\end{abstract}

Keywords. stars: formation; stars: brown dwarfs; planetary systems; instrumentation: adaptive optics

\section{Introduction}

Over the past 25 years, huge progress has been made in our understanding of planetary systems around other stars. The IRAS mission identified the stars with circumstellar dust, defining the target lists for studies of star and planet formation. Kinematic studies by millimeter interferometers demonstrated that these dust clouds were truly disks. The Hubble Space Telescope and adaptive optics on new 8-10m telescopes have imaged the detailed disk structure, and are beginning to find low mass companions to young stars. Brown dwarfs, the Kuiper Belt, and extrasolar planets were all discovered through patient survey work. The pace of progress will accelerate through projects such as the Spitzer Space Telescope, Herschel, and ALMA - and, in the next decade, the development of Extremely Large Telescopes (ELTs). In the following contribution, I outline the key contributions ELTs can make to studies of star formation, circumstellar disks, and extrasolar planets.

\section{Disks in our Solar System}

The study of asteroids and Kuiper Belt Objects (KBOs) is part of the larger story of planet formation. Wide-field synoptic surveys with the upcoming Pan-STARRS and 
LSST facilities will greatly expand the number of known moving objects down to $V=25$. While existing telescopes are sufficient to characterize their orbits and broad-band colors, ELTs will be needed to determine their chemical make-up, sizes, and masses.

KBOs show a remarkable diversity in their broad-band colors that holds clues to their surface characteristics, formation, and dynamical evolution. In a few bright cases nearinfrared spectra have been used to relate colors to the presence of various ices of $\mathrm{CO}, \mathrm{CO}_{2}$, $\mathrm{CH}_{4}$, and $\mathrm{N}_{2}$ (Cruickshank et al. 2006). However, most KBOS are too faint for moderate resolution spectroscopy with existing telescopes. ELTs will enable a broad assessment of compositional differences within the Kuiper Belt, and comparisons to long-period comets.

With Laser Guide Star (LGS) adaptive optics, a 30m ELT can resolve the sizes of KBOs with $\mathrm{d}>500 \mathrm{~km}$ at $50 \mathrm{AU}$ distance, main belt asteroids with $\mathrm{d}>20 \mathrm{~km}$ at $2 \mathrm{AU}$ distance, and Earth-crossing asteroids with $\mathrm{d}>1 \mathrm{~km}$ at $0.1 \mathrm{AU}$ distance. Size measurements permit an object's albedo and surface temperature to be derived from reflectance data. They are especially needed for KBOs, where few objects have proven to be detectable in the thermal infrared. More than a dozen main belt asteroids are now known to be binary. Surprisingly, the binary frequency of KBOs is estimated to be as high as $10 \%$. Binary asteroids and KBOs are extremely important objects: through measurements of orbital periods, the system mass can be derived from Kepler's laws. In combination with size measurements, the object's density can then be estimated - a major constraint on its composition and internal structure. ELTs will enable searches for companions that are fainter, and at smaller orbital separations, than those detected with current facilities. Their astrometric accuracy will improve determinations of binary orbits, perhaps to the point where tidal effects and higher-order gravitational moments can be assessed.

\section{Star formation}

Age dating of young stars is done through comparisons to evolutionary tracks in the HR diagram. These tracks have considerable uncertainty, particularly at ages $<10 \mathrm{Myr}$ (Hillenbrand \& White 2004). Accurate calibration of pre-main sequence (PMS) evolutionary tracks is essential to studies of the initial mass function and its dependence on protostellar environment, and to studies of disk evolution. The key to progress is through studies of PMS binaries, where dynamical masses can be measured. A 30m ELT will advance the field by tracking the orbits of binaries with separations as small as $7 \mathrm{AU}$ $(\mathrm{d}=140 \mathrm{pc})$, discovering previously unknown faint companions, and by providing high resolution spectroscopy (and thus $\mathrm{T}_{\text {eff }}$ values) for the individual components. These applications will require LGS AO with good Strehl ratios, and in many cases, coronagraphic spectroscopy.

The youngest stars commonly possess collimated outflows energized by accretion from circumstellar matter. These flows create high velocity shocks that are seen as emission line nebulae called Herbig-Haro objects. They are nearby, low velocity (few hundred $\mathrm{km} \mathrm{s}^{-1}$ ) examples of astrophysical jets seen in quasars and radio galaxies. Variability in the jet velocity and perhaps the mass flow rate produces multiple shocks within the flow and major bowshocks in the surrounding medium, tracing the time history of the accretion process. In the near-infrared, lines of $\mathrm{H}_{2}$ and [Fe II] are used to study the flow structure. ELTs equipped with LGS adaptive optics will provide imaging and spectroscopy at spatial resolutions of $2 \mathrm{AU}$ in nearby star-forming regions. This will enable detailed studies of jet collimation near the central source, entrainment of ambient cloud material at the jet periphery, and spectroscopic studies of jet velocity structure. Proper motions in the flows will be detectable in a time baseline of only a few months. These datasets will be highly valuable toward the goals of understanding the relative contributions of disk winds and 
stellar winds to outflows, and confirming helical velocity structures reported in YSO jets from Hubble Space Telescope data.

Planet formation takes place within the circumstellar disks of young stars. Studies of disk structure and composition, their dependence on environment, and how they evolve with time are crucial to understanding how the process takes place. Key issues include the evolution of dust particles from ISM sizes to planetesimals, the chemistry of ices and disk gas, whether planets form while the star is still accreting from the disk, the extent to which protoplanets clear disk holes and gaps, and the timescale for disk gas dissipation. Before ELTs come on line, results from the Atacama Large Millimeter Array (ALMA) are likely to make major contributions to these science topics. The unique applications for ELTs will be disk studies that require high spatial and spectral resolution in the nearinfrared. These include spectroscopy of warm gas in the inner disk, multicolor imaging in scattered light to search for evolution/depletion in the small grain population, resolving disk central holes inferred to exist from spectral energy distributions, and direct detection of hot young protoplanets within disks. A particularly promising case is the nearby $\mathrm{T}$ Tauri star TW Hydrae, which has a face-on disk massive enough to form a planetary system. This disk is inferred to have a central hole of $4 \mathrm{AU}$ ( 80 mas) radius, which should be resolvable with AO coronagraphy on a 30m ELT. Dynamical clearing of the inner disk by a protoplanet or proto brown dwarf has been suggested as the origin of the hole (Calvet et al. 2002); such an object should be detectable with a contrast $>10^{-5}$. Surveys of a large sample of disk-bearing young stars have the potential to reveal numerous examples of disk/planet interactions very early in the life of a planetary system.

\section{Exoplanet: indirect detections}

As of this writing, 170 extrasolar planets have been detected indirectly by the radial velocity (RV) technique (Schneider 2005). These planets have Msin $i$ ranging from 7 Earth masses to 17 Jupiter masses $\left(M_{J}\right)$, and orbital periods from 2 days to 12 years. Eight objects are seen to transit their parent stars, allowing the planetary masses and radii to be precisely determined. Planets are observed to be more common at smaller masses $\left(d N / d M \propto M^{-1}\right)$, at longer orbital periods, and higher host star metallicities (Marcy et al. 2005). These accumulated results represent a major new field of astrophysics, and one of the more prominent scientific accomplishments of the past decade.

Our knowledge of RV planets is currently limited by the few $\mathrm{ms}^{-1}$ velocity precision of current facilities, and by the finite time baseline over which stellar radial velocities have been monitored. Ongoing surveys target a few thousand nearby FGK stars, using 2-10m telescopes equipped with high resolution spectrographs and gas absorption cells as wavelength references. The typical target star has $V<8$, but a subsample of $\sim 200$ fainter $\mathrm{M}$ stars is also being studied at degraded velocity precision. In the next few years, new facilities are expected to deliver radial velocity precision down to $1 \mathrm{~m} \mathrm{~s}^{-1}$. However, it should be noted that velocity variations of $5-10 \mathrm{~m} \mathrm{~s}^{-1}$ naturally occur in these targets due to stellar activity. Current observations are therefore limited by astrophysical noise, and the accuracy of instrumental calibration, not by photon statistics (whose contribution to the uncertainty is typically only at the $20 \mathrm{~cm} \mathrm{~s}^{-1}$ level; Udry et al. 2006).

ELTs will play several roles in future indirect detections of extrasolar planets. They will extend the reach of highest precision RV observations down to $V=12$ or so, allowing much larger samples of all stellar types be monitored for planets. One application of this capability will be in follow-up studies to the numerous surveys for transiting planets being carried out now on the ground, and to be carried out with the COROT and Kepler space missions scheduled for launch this decade. It is expected that these projects will discover 
hundreds of additional stars with transiting planets. The majority of these will be faint, so ELTs will be the facilities of choice for high resolution spectroscopy to measure the radial velocity curves and derive precise planetary masses. ELTs should also help in the quest to detect molecular features in the planetary atmospheres, which will be sought in near-IR spectra taken during transit. Another application of the increased limiting magnitude for RV surveys with ELTs will be M stars, where the small stellar mass results in a larger reflex motion, and thus better sensitivity to low-mass planets. For an M5 star, $1 \mathrm{~ms}^{-1}$ precision would just allow detection of a 2 Earth mass planet in the liquid water habitable zone (orbital semi-major axis of $0.1 \mathrm{AU}$ ). Finally, seeing-limited relative astrometry over 2 arcmin fields of view could be capable of $30 \mu$ as RMS measurements on a $30 \mathrm{~m}$ telescope (Pravdo \& Shaklan 1996). This would allow astrometric detection of Neptune-mass planets orbiting $2 \mathrm{AU}$ from nearby M stars.

\section{Brown dwarfs}

With masses intermediate between giant planets and low-mass stars, brown dwarfs derive their luminosity from residual heat of formation. The temperature of a brown dwarf depends both on its initial heat content (and thus its mass), and how long it has been radiating that heat away to space. An extensive literature now exists predicting brown dwarf evolutionary tracks and detailed emergent spectra from the optical through the mid-infrared. The cooler brown dwarfs, spectral class " $\mathrm{T}$ " ( $\mathrm{T}_{\text {eff }}<1200 \mathrm{~K}$ ), feature prominent methane absorption features. About $60 \mathrm{~T}$ dwarfs are now known: most are isolated field objects discovered by the 2MASS or Sloan sky surveys. A handful of T dwarfs have also been found as companions to nearby stars. The coolest known $\mathrm{T}$ dwarf has an effective temperature of $700 \mathrm{~K}$. Objects with cooler temperatures are thought to exist, but are unknown observationally above the temperature of our own Jupiter, $\mathrm{T}_{\text {eff }}=$ $120 \mathrm{~K}$. When finally found, these hypothesized "Y dwarfs" will be the first examples of astrophysical polytropes in the temperature range 200-600 K.

ELTs can be expected to play several important roles in brown dwarf research. Upcoming infrared surveys (the groundbased UKIDSS, and NASA WISE space mission) should discover a faint population field brown dwarfs, hopefully including many Y dwarf candidates. ELTs will be the facilities of choice for follow-up spectroscopy of these targets at near-infrared wavelengths. The spectra they provide, in conjunction with model atmospheres, will allow the $\mathrm{T}_{\text {eff }}$, composition, surface gravity, and role of clouds and hazes to be determined. Many brown dwarfs are known to be binaries. LGS AO on ELTs can be expected to discover binary brown dwarfs at smaller separations than previously possible. In the binaries that are identified, LGS imaging will provide accurate astrometric tracking of the orbits, and thus dynamical masses. In combination with spatially resolved high resolution spectroscopy at $\sim 100$ mas separations, these measurements will be highly valuable for calibrating theoretical brown dwarf evolutionary tracks.

Finally, ELTs should be extremely capable machines for brown dwarf companion searches to nearby stars. Very few brown dwarf companions have been found at orbital radii $<5 \mathrm{AU}$, leading to the term "brown dwarf desert". However, they are seen at wide separations $>300 \mathrm{AU}$, where they may be almost as common as stellar companions (Gizis et al. 2001). The bridge between these two discordant results must be built through searches at intermediate separations. Such companion searches are part of the larger story of exoplanet direct imaging. 


\section{Exoplanets: direct detections}

Extrasolar planets can be divided into three observational categories: (1) "Roasters", which orbit very close to their parent star, and whose strong infrared emission is driven by the intense stellar radiative input; (2) Young/massive giant planets and brown dwarfs, whose luminosity is dominated by residual internal heat of formation; and (3) "Cold" planets with little internal heat, and seen primarily by reflected starlight. Roasters present the easiest contrast challenge $\left(\sim 10^{-4}\right)$, but all known examples lie within a few mas of their host stars, and are thus not accessible to direct imaging, even with next generation ELTs. Young/massive planets are expected to appear at contrasts $>10^{-7}$, and at range of angular separations that will make them excellent targets for future ELTs. Indeed, initial steps in the direct detection of these objects have already taken place. Cold planets present the most difficult challenge (contrast $<10^{-8}$ ), and their detection at subarcsecond separations is unlikely to be possible through the atmosphere (see below).

Two groups have now reported adaptive optics direct imaging of very low-mass companions to very young stars. 2MASS1207 b (Chauvin et al. 2005) and GQ Lupi b (Neuhauser et al. 2005) have been clearly established as common-proper motion companions to their primaries: a brown dwarf and a $\mathrm{T}$ Tauri star respectively. The companion masses $\left(5 \mathrm{M}_{J}\right.$, and probably $\left.20 \mathrm{M}_{J}\right)$ are estimated from model evolutionary tracks, and depend sensitively on both the model details and on knowledge of the ages \& distances of the primaries. Star-forming regions and nearby young associations (TW Hydrae, $\beta$ Pictoris, Tucanae, etc.) are being surveyed for companions by a number of groups. Additional detections of objects like these can be expected. While these detections are interesting, they are only a small step toward the goal of imaging planetary systems like our own. The 2MASS1207 and GQ Lupi companions lie at large projected separations $\left(0.8^{\prime \prime} ; 50\right.$ and $100 \mathrm{AU}$ respectively), have extremely young ages of 8 and $1 \mathrm{Myr}$, and are seen at the easily-achieved image contrast of only $\sim 10^{-2}$.

Direct detection of extrasolar planets at separations $<10 \mathrm{AU}$, and orbiting nearby main sequence stars with ages of a few Gyrs, is a key goal for observational astronomy in the coming decades. Direct detection enables the spectral studies needed to understand planetary composition and atmospheric structure, can resolve orbital orientation angles that are indeterminate from radial velocity measurements, and can discover planets in regions of phase space (long-period orbits; lower planetary masses) that are inaccessible to indirect measurements. High contrast imaging detections are limited to companion separations outside a certain inner working angle (IWA) and above a certain limiting contrast, with the latter being a strong function of angular separation. The current state-of-the-art for image contrast with adaptive optics on $8-10 \mathrm{~m}$ telescopes is a factor of $10^{-5}$ at $0.5^{\prime \prime}$ separation, achieved with simultaneous differential imaging at the VLT (Biller et al. 2004). How much better is high contrast imaging likely to be with future ELTs? This key question is now considered in some detail.

An important feature of future Extreme Adaptive Optics (ExAO) systems is that their point spread functions (PSFs) include a dark hole region of maximum starlight suppression around the target star. The dark hole radius is determined by the number $\mathrm{N}$ of deformable mirror actuators across the primary mirror $\left(\theta_{\text {rad }}=N \lambda / 2 D\right)$, and its depth by how accurately atmospheric wavefront errors can be corrected. Today's AO systems have very small dark holes, and suffer from large wavefront errors $\sim 200 \mathrm{~nm}$. Two next generation high contrast AO systems, the VLT Planet Finder (Beuzit et al.) and Gemini ExAOC (Macintosh et al.), aim to provide $10^{-6}$ contrast within a $\sim 1^{\prime \prime}$ dark hole radius at $H$-band. First results from these two projects are expected in 2010, which could be too late to incorporate lessons learned into first generation ELT instruments. 
Table 1. Speckle contrast considerations for extreme adaptive optics

\begin{tabular}{cccccc}
\hline Contrast & $\begin{array}{c}\text { Coherent } \\
\text { wavefront } \\
\text { error }^{1}\end{array}$ & $\begin{array}{c}\text { RMS } \\
\text { wavefront } \\
\text { error }^{2}\end{array}$ & $\begin{array}{c}\text { RMS } \\
\text { pathlength } \\
\text { error }^{3}\end{array}$ & $\begin{array}{c}\text { Reduced } \\
\text { coherence }^{\text {time }}{ }^{4}\end{array}$ & $\begin{array}{c}\text { Guidestar } \\
H \\
\text { magnitude }^{5}\end{array}$ \\
\hline $10^{-6}$ & $\lambda / 4,400$ & $\lambda / 88$ & $18.7 \mathrm{~nm}$ & $1.07 \mathrm{~ms}$ & 6.9 \\
$10^{-7}$ & $\lambda / 14,000$ & $\lambda / 280$ & $5.7 \mathrm{~nm}$ & $0.34 \mathrm{~ms}$ & 3.2 \\
$10^{-8}$ & $\lambda / 44,000$ & $\lambda / 880$ & $1.9 \mathrm{~nm}$ & $0.11 \mathrm{~ms}$ & -0.6 \\
$10^{-9}$ & $\lambda / 140,000$ & $\lambda / 2,800$ & $0.6 \mathrm{~nm}$ & $0.03 \mathrm{~ms}$ & -4.3 \\
$10^{-10}$ & $\lambda / 440,000$ & $\lambda / 8,800$ & $0.2 \mathrm{~nm}$ & $0.01 \mathrm{~ms}$ & -8.1 \\
\hline
\end{tabular}

1 Amplitude of a single sinusoidal phase ripple across the entrance pupil, whose corresponding focal plane image speckle would present the contrast to the central star specified in column 1.

${ }_{2}$ Reduced phase error after averaging over 2500 incoherent modes. This corresponds to a $10^{4}$ actuator AO system, which, on a $30 \mathrm{~m}$ telescope, would yield a high contrast dark field $0.57^{\prime \prime}$ in radius at $H$-band.

${ }^{3}$ For $H$-band science observations

4 The time over which the phase of the atmospheric wavefront changes by less than the RMS error values given in columns $3 \& 4$, assuming a standard coherence time of $15 \mathrm{~ms}$ at $H$-band.

${ }^{5}$ Guidestar brightness needed to completely sense the wavefront to the accuracy given in column 4, within the reduced coherence time of column 5, for $10^{4}$ subapertures on a $30 \mathrm{~m}$ telescope. A noiseless infrared interferometric wavefront sensor with $50 \%$ throughput operating at $20 \%$ bandwidth is assumed.

There is much speculation about what levels of high contrast imaging might be possible with future ELTs. A limit, or at least a break point in their performance, can be derived from considerations of wavefront stability requirements, atmospheric stability timescales, and the photon fluxes available from natural guidestars. The depth of the high contrast dark hole is set by residual speckles from uncorrected wavefront errors. Unfortunately these speckles are neither static (in which case they could be calibrated out) nor fully stochastic (in which case they could be averaged over); they evolve unpredictably on a variety of timescales governed by the atmospheric turbulence profile, and by vibration, flexure, and thermal changes in the telescope and instrument. The AO system corrects these speckles in real-time, but only to a certain accuracy. Stronger speckle suppression requires wavefront corrections to higher accuracies and on faster timescales, as shown in Table 1. Eventually a contrast limit is reached below which there are not enough guidestar photons to suppress the residual speckles any further. For a solar twin at $10 \mathrm{pc}(H=3.0)$, this contrast limit is $10^{-7}$. If we require a fixed dark hole outer radius in arcsec, this speckle contrast limit becomes insensitive to the telescope aperture D: larger apertures require a larger number $\mathrm{N}$ of subapertures to be sensed and controlled by the ExAO system, leaving the number of photons per subaperture (and thus these magnitude/contrast limits) unchanged.

Detecting point sources fainter than the residual speckles is possible if a reference PSF can be obtained before the wavefront changes by more than the limits given in Table 1, column 4. In practice this means simultaneous imaging at adjacent wavelengths: residual speckles will shift position with wavelength, whereas an actual companion object will not. Experience with simultaneous differential imaging at the VLT has shown that objects as much as $10 \times$ fainter than the speckles can be recovered (B. Biller, personal communication); and a similar limit is seen for PSF subtraction at $r<2^{\prime \prime}$ with the Hubble Space Telescope (Krist 2004). This additional factor of ten suggests an ultimate groundbased contrast limit of $10^{-8}$, for surveys of nearby solar-type stars.

It has been suggested that terrestrial planets, with contrasts of $10^{-10}$, might be detectable with future large ELTs in integration times of a few hours. While photon 
statistics might allow such detections, the systematic errors of residual speckles are unlikely to. As discussed above, wavefront control considerations will limit speckle suppression to the $10^{-7}$ contrast level. To recover the signal of a terrestrial planet, the variable (but incompletely stochastic) field of residual speckles would need to be subtracted away to an accuracy of $0.1 \%$. This requirement is a hundred times beyond what has been achieved to date by any high contrast imaging system, on the ground or in space. It may even be precluded by flat fielding uncertainties. Instrument development work is needed to explore whether the current $10 \%$ limit to speckle subtraction accuracy can be improved upon. AO imaging of bright stars against dense galactic starfield backgrounds ("The Deneb Deep Field") would be ideal datasets for testing the limits of speckle subtraction and source recovery. In the absence of progress in this area, it would be very risky to base ELT science cases on the ability to detect planets at contrasts below $10^{-8}$.

The above considerations argue for a limiting contrast of $10^{-8}$ for extreme adaptive optics imaging of nearby solar-type stars with ELTs. Achieving this contrast performance three orders of magnitude beyond the current state of the art - will require significant capital and human investments. Instrumental requirements include larger deformable mirrors, low-noise focal plane wavefront sensors, AO operation at frequencies of many $\mathrm{KHz}$, major increases in real-time computing capability, integral field units as coronagraphic backends, careful attention to telescope mechanical stability, control of stray light, and strong suppression of diffraction (which is greatly facilitated by minimizing segmentation, as in the GMT design concept). One or more generations of ExAO systems, beyond the currently funded VLT and Gemini "planet finders", will likely be needed to push these developments before $10^{-8}$ contrast performance could be realized on next-generation ELTs.

If ExAO systems capable of this contrast performance can be built, what types of companion objects might they be able to access? The contrast needed to detect extrasolar giant planets is a strong function of wavelength and orbital separation. This is illustrated in Fig. 1, from models by Burrows et al. (2004). Like brown dwarfs, the spectra of extrasolar giant planets are strongly cut by methane absorption features. Flux "peaks" at 1.0, $1.2,1.6,2.1$ and $4.5 \mu \mathrm{m}$, representing gaps between methane absorptions, establish preferred wavelengths for direct imaging searches. Each wavelength has its own advantages and disadvantages for direct detection.

The best intrinsic star/planet contrast $\left(>10^{-6}\right)$ is found at $M$-band. $M$-band also has the advantage that adaptive optics systems provide their best Strehl ratios (and thus best contrast performance) at the longest wavelengths. However, even for a $30 \mathrm{~m}$ telescope, high sky backgrounds will limit detections to objects brighter than apparent magnitude $M=19$ in a full night of integration. According to the Burrows et al. models, for a star at $10 \mathrm{pc}$ distance, this brightness limit is equivalent to the flux density from (1) a warm 5 Gyr age, 13 Jupiter mass brown dwarf; (2) a warm 1 Gyr age, 5 Jupiter mass planet; or (3) a cold Jupiter-size planet in an orbit $\leqslant 1$ AU from a G2 star. RV surveys have largely explored the population of giant planets inside the $1 \mathrm{AU}$ horizon around nearby stars. None are known that have both orbits inside 1 AU, and maximum elongations greater than the $0.13^{\prime \prime}$, the inner working angle (IWA) of a $30 \mathrm{~m}$ telescope at $\lambda=4.7 \mu \mathrm{m}$ (IWA $=4 \lambda / D$ for a Lyot coronagraph). However, companions with significant internal heat (mass $>5 \mathrm{M}_{J}$ ) will be detectable outside this IWA, and should be the subjects of intensive searches. Some early results can be expected from the Large Binocular Telescope, for $M<17$.

Better inner working angles, much lower sky backgrounds, and higher contrast sensitivity are available in the near infrared. Of the near-IR bands, $H$ probably offers the best combination of small inner working angle ( $\sim 40$ mas for a $30 \mathrm{~m}$ telescope), sensitivity to 


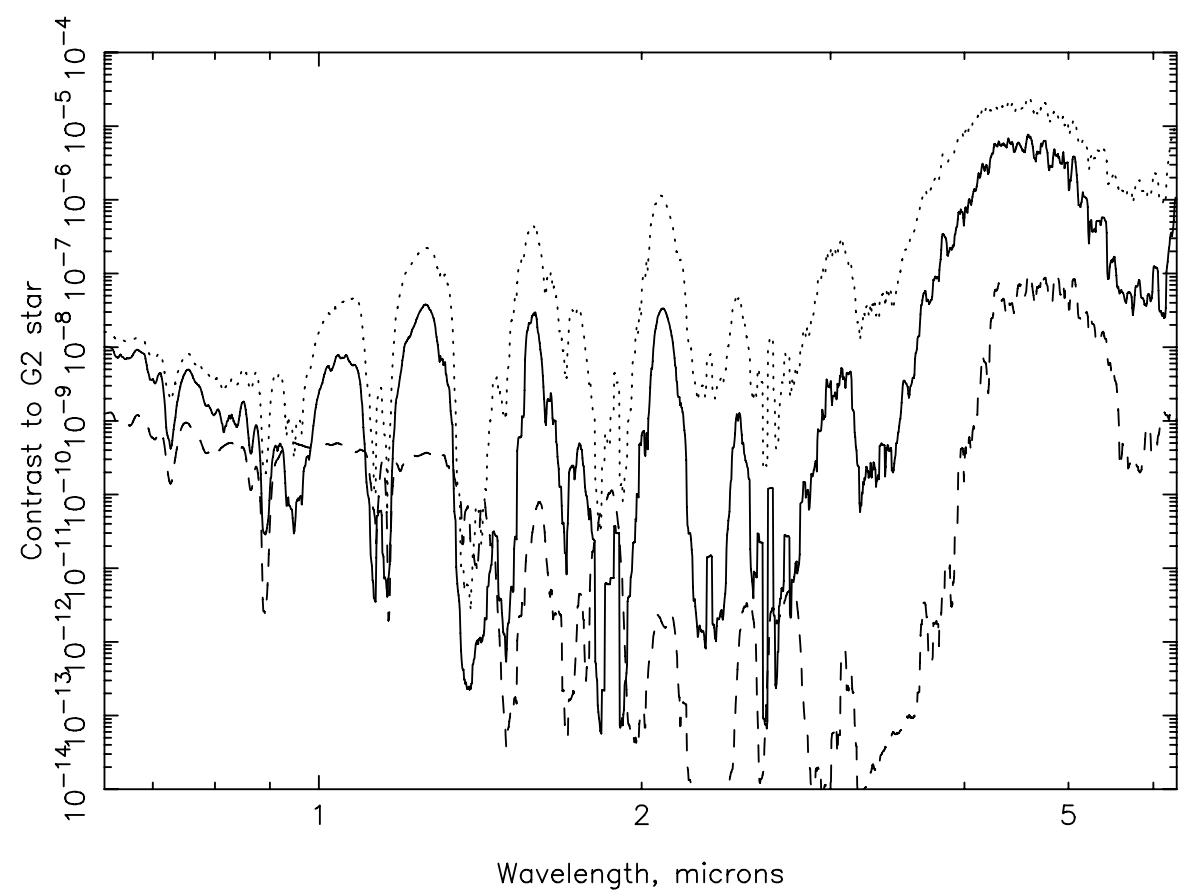

Figure 1. Predicted contrast spectra of extrasolar jovian planets relative to a G2 V parent star, from Burrows, Sudarsky \& Hubeny (2004). The dotted, solid, and dashed lines show model spectra of a $1 \mathrm{M}_{J}$ planet located $0.5,1$, and $4 \mathrm{AU}$ from the star. A "cold" planet is assumed, i.e. the planet's thermal state is dominated by stellar energy input, with minimal internal heat. Reflected light and thermal emission are both included, with the latter corresponding to effective temperatures of 430,300 , and $150 \mathrm{~K}$ respectively.

giant planets over a wide range of temperatures, good adaptive optics performance, and the potential to reach contrasts of $10^{-8}$. Comparing these limits to the model spectra in Fig. 1, the predicted $H$-band contrast of cold giant planets is greater than $10^{-8}$ for orbits with $r \leqslant 1$ AU around a G2 star. The Extrasolar Planets Encyclopedia (Schneider 2005) currently lists three known giant planets whose maximum elongations exceed 40 mas, but which still have orbital semi-major axes $<1 \mathrm{AU}$; see the top section of Table 2.

In addition to the above "cold" planets, RV surveys have detected massive planets whose large reservoirs of internal heat should render them brighter than the ExAO $H$ band limiting contrast of $10^{-8}$. The emission from a massive planet depends on its mass, age, and radiative input from the star; so the threshold for their detectability is complex. A simplified dividing line is $8 \mathrm{M}_{J}$, above which the models of Burrows et al. (2004) predict an $H$-band contrast $>10^{-8}$ for a 5 Gyr age G2 star. Nine RV planets are currently known with $\mathrm{Msin} i>8 \mathrm{M}_{J}$ and maximum elongations greater than 40 mas (Schneider 2005); see the bottom section of Table 2 . One (HD $39091 \mathrm{~b}$ ) has a particularly favorable contrast and maximum elongation, and if the models are correct, could be the first RV planet to be directly imaged. The last two sources on the list may also be detectable at $5 \mu \mathrm{m}$. Ongoing RV surveys should add to this list by the time ELTs with $10^{-8}$ contrast ExAO systems come on-line.

If the necessary instrumentation can be developed, a 30m ELT should be capable of directly detecting the dozen extrasolar planets listed in Table 2. These targets should then be highest-priority for high contrast imaging with ELTs. In addition, an imaging survey of a large number of nearby stars should uncover numerous self-luminous planetary 
Table 2. Known radial velocity planets with predicted

$H$-band contrast $>10^{-8}$, max elongation $>40$ mas:

ExAO on a $30 \mathrm{~m}$ ELT

\begin{tabular}{|c|c|c|c|c|c|}
\hline $\begin{array}{l}\text { Planet } \\
\text { name }\end{array}$ & $\begin{array}{l}\text { Stellar } \\
\text { spectral } \\
\text { type }\end{array}$ & $\begin{array}{r}\text { Planet } \\
\text { mass } \\
\times \sin i\end{array}$ & $\begin{array}{c}\text { Orbital } \\
\text { semi-major } \\
\text { axis }\end{array}$ & $\begin{array}{l}\text { Maximum } \\
\text { Elongation }\end{array}$ & Notes \\
\hline HD $142 \mathrm{~b}$ & G1 & $1 \mathrm{M}_{J}$ & $0.98 \mathrm{AU}$ & 48 mas & \\
\hline HR $810 \mathrm{~b}$ & G0 & $2 \mathrm{M}_{J}$ & $0.91 \mathrm{AU}$ & 59 mas & \\
\hline$v$ And $\mathrm{c}$ & F8 & $2 \mathrm{M}_{J}$ & $0.83 \mathrm{AU}$ & 62 mas & $10^{-7}$ contrast, $\mathrm{SBH} 03$ \\
\hline HIP 75458 b & $\mathrm{K} 2$ & $9 \mathrm{M}_{J}$ & $1.34 \mathrm{AU}$ & 43 mas & \\
\hline HD 136118 b & F9 & $12 \mathrm{M}_{J}$ & $2.30 \mathrm{AU}$ & 44 mas & \\
\hline HD 141937 b & G2 & $10 \mathrm{M}_{J}$ & $1.52 \mathrm{AU}$ & 45 mas & \\
\hline HD 33564 b & $\mathrm{F} 6$ & $8 \mathrm{M}_{J}$ & $1.10 \mathrm{AU}$ & $52 \mathrm{mas}$ & \\
\hline HD 30177 b & G8 & $9 \mathrm{M}_{J}$ & $3.90 \mathrm{AU}$ & 70 mas & \\
\hline HD $168443 \mathrm{c}$ & G5 & $17 \mathrm{M}_{J}$ & $2.90 \mathrm{AU}$ & 87 mas & brown dwarf \\
\hline HD $38529 \mathrm{c}$ & G4 & $13 \mathrm{M}_{J}$ & $3.68 \mathrm{AU}$ & 87 mas & brown dwarf \\
\hline HD $33636 \mathrm{~b}$ & G0 & $9 \mathrm{M}_{J}$ & $3.56 \mathrm{AU}$ & $124 \mathrm{mas}$ & $5 \times 10^{-7}$ contrast, BSH04 \\
\hline HD $39091 \mathrm{~b}$ & G1 & $10 \mathrm{M}_{J}$ & $3.29 \mathrm{AU}$ & $160 \mathrm{mas}$ & $10^{-6}$ contrast, BSH04 \\
\hline
\end{tabular}

Top section: "Cold" planets lacking major internal heat sources

Bottom section: Massive planets expected to have strong internal heat sources

companions at contrasts $>10^{-8}$, and whose orbital periods are too long for them to have been detected yet in RV surveys. For the objects where Msin $i$ is known, the imaging detections will resolve the unknown orbit inclination, and refine the mass values. This will allow the observed planetary fluxes, in combination with stellar age estimates, to provide critical tests for model evolutionary tracks for giant planet and brown dwarfs. Spectral characterization will be crucial for understanding the atmospheric structure and composition, but would be limited to narrow wavelength windows if $\mathrm{CH}_{4}$ opacity dominates the spectrum as current models suggest.

\section{Acknowledgements}

I would like to thank Richard Dekany, Bruce Macintosh, Wes Traub, and John Trauger for numerous discussions of high contrast adaptive optics. Thanks also to the meeting organizers, and particularly Mark McCaughrean, for the opportunity to present this material. Funding for this work was provided by NASA to the Jet Propulsion Laboratory, California Institute of Technology.

\section{References}

Biller, B.A. et al. 2004 SPIE 5490, 389

Burrows, A., Sudarsky, D. \& Hubeny, I. 2004, ApJ 609 416, (BSH04)

Calvet, N. et al. 2002 ApJ 568, 1008

Cruickshank, D.P. et al. 2006, in: B. Reipurth, D. Jewitt \& K. Keil (eds.), Protostars and Planets $V$, Univ. of Arizona Press, Tucson

Chauvin, G. et al. 2005, A\&\&A 438, L25

Gizis, J.E. et al. 2001, ApJ 551, L163

Hillenbrand, L.A. \& White, R.J. 2004, ApJ 604, 741

Krist, J.E. 2004, SPIE 5487, 1284 
Marcy, G. et al. 2005, P.Th.P.S. 158, 24

Neuhauser, R. et al. 2005, A\&A 435, L13

Pravdo, S.H. \& Shaklan, S.B. 1996, ApJ 465, 264

Schneider, J. 2005 http://www.obspm.fr/planets catalog-main

Sudarsky, D., Burrows, A. \& Hubeny, I. 2003, ApJ 588, 1121 (SBH03)

Udry, S., Fischer, D. \& Queloz, D. 2006, in: B. Reipurth, D. Jewitt \& K. Keil (eds.), Protostars and Planets $V$, Univ. of Arizona Press, Tucson

\section{Discussion}

BURLEIGH: White dwarfs are an excellent target for companion searches by direct imaging, e.g. brown dwarfs and 400K polytropes, because of the huge contrast gain, known age and distance, and white dwarfs are remnants of main sequence stars.

STAPLEFELDT: Thank you for pointing this out!

SACKETT: A comment more that a question. If we can find many transiting systems which won't be done with ELTs - I believe ELT will be able to distinguish transiting planets from grazing binaries or blended binaries much more easily than relying on a radial velocity measurements. This could be done by using all of the spectrum and making broadband measurements of colour changes during the course of the transit... A transiting small planet creates a colour signature, as it crosses a limb-darkened star, of about a milli-mag, which should be drop-dead easy with an ELT. The signature is much different for the pretenders.

StAPELfEldT: Do you assume the stellar companion has a different colour than the primary?

SACKETT: No the colour effect comes from limb darkening.

Hook: Has the 'Q' factor you described been calculated for the case of an earth-like planet and a telescope with $\mathrm{D}>30 \mathrm{~m}$ ?

StApelfeldt: I didn't show it, but $\mathrm{Q}$ goes as $\mathrm{D}^{2}$. So extrapolating from $Q=10^{-4}$ for observing an Earth on a $30 \mathrm{~m}$, to $Q=10^{-3}$ for a $100 \mathrm{~m}$ aperture.

ZINNECKER: You did not mention high-mass star formation, only low-mass star formation. I'd just like to stress that there is also an excellent science case for the former. I will discuss some possible studies of massive star-birth in my talk on Thursday. 